Revue internationale P.M.E.

Économie et gestion de la petite et moyenne entreprise

\title{
Les réseaux de l'innovation dans les PMI : illustration sur le cas de l'Alsace
}

\section{Jean-Alain Héraud et Constantin Nanopoulos}

Volume 7, numéro 3-4, 1994

URI : https://id.erudit.org/iderudit/1008424ar

DOI : https://doi.org/10.7202/1008424ar

Aller au sommaire du numéro

Éditeur(s)

Presses de l'Université du Québec

ISSN

0776-5436 (imprimé)

1918-9699 (numérique)

Découvrir la revue

Citer cet article

Héraud, J.-A. \& Nanopoulos, C. (1994). Les réseaux de l'innovation dans les PMI : illustration sur le cas de l'Alsace. Revue internationale P.M.E., 7(3-4),

35-86. https://doi.org/10.7202/1008424ar
Résumé de l'article

Cet article examine la notion de réseau territorialisé d'innovation à la lumière d'une enquête menée auprès d'un échantillon de 161 entreprises de moins de 500 salariés, représentatif du tissu industriel alsacien. On met en évidence la variété des réseaux contribuant au processus d'innovation des firmes et surtout la forte disparité de leurs projections géographiques. Aussi bien pour les réseaux de partenariat formel (accords) que pour les relations informelles, la proximité géographique ne semble pas toujours jouer un rôle de premier plan ou, du moins, les effets de proximité sont variables selon le type d'interaction que l'on considère. D'où la difficulté de parler d'un territoire ou d'un milieu innovant proprement alsacien. L'idée d'un réseau transfrontalier dans le bassin du Rhin supérieur n'est pas non plus confortée par l'observation, sauf sur des points très précis. 


\title{
Les réseaux de l'innovation dans les PMI: illustration sur le cas de l'Alsace
}

\author{
Jean-Alain HÉRAUD* \\ Université Louis-Pasteur, Strasbourg \\ Constantin NANOPOULOS** \\ Université Robert-Schuman, Strasbourg
}

\section{MOTS CLÉS}

\author{
Innovation - PME - Région - Réseau - Localisation \\ Industrie - Partenariat
}

* Jean-Alain Héraud est professeur de sciences économiques à l'Université Louis-Pasteur (Strasbourg 1). Il est actuellement directeur du BETA, unité de recherche en économie et gestion associée au CNRS (URA 1237). L'économie industrielle et la théorie de l'innovation constituent l'une des spécialités majeures du laboratoire. Les publications et activités de recherche propres à l'auteur dans ce cadre sont l'analyse des réseaux technologiques régionalisés, la prospective technologique, l'économie de la propriété intellectuelle et les indicateurs de sciences et techniques. Adresse : BETA, Université Louis-Pasteur, 38, boulevard d'Anvers, F-67000 Strasbourg, France.

** Constantin Nanopoulos est maître de conférences à l'IECS-Strasbourg de l'Université Robert-Schuman (Strasbourg) 3), membre du CESAG et collaborateur du BETA (Université Louis-Pasteur). Docteur de $3^{\mathrm{e}}$ cycle en mathématiques, directeur pédagogique à l'IECS et vice-président de l'Association information et management, il est l'auteur de plusieurs articles dans les domaines du management international et de la gestion industrielle. Adresse: IECS, Université Robert-Schuman, 47, avenue de la Forêt Noire, F-67000 Strasbourg, France. 


\title{
RÉSUMÉ
}

Cet article examine la notion de réseau territorialisé d'innovation à la lumière d'une enquête menée auprès d'un échantillon de 161 entreprises de moins de 500 salariés, représentatif du tissu industriel alsacien. On met en évidence la variété des réseaux contribuant au processus d'innovation des firmes et surtout la forte disparité de leurs projections géographiques. Aussi bien pour les réseaux de partenariat formel (accords) que pour les relations informelles, la proximité géographique ne semble pas toujours jouer un rôle de premier plan ou, du moins, les effets de proximité sont variables selon le type d'interaction que l'on considère. D'où la difficulté de parler d'un territoire ou d'un milieu innovant proprement alsacien. L'idée d'un réseau transfrontalier dans le bassin du Rhin supérieur n'est pas non plus confortée par l'observation, sauf sur des points très précis.

\begin{abstract}
In this paper, we examine the notion of "territorialised" innovation network. After reviewing the literature on the subjecte, we try to confront the prevailing theories and concepts with an industrial inquiry on the Alsatian industry (based on a representative sample of 161 SMEs). We observe a wide variety of networks leading to innovation. The geographical projections of these networks are particularly scattered in space. For informal networks as well as for formal ones (cooperative agreements), we conclude that proximity does not systematically play an important role in the innovative activities of firms, although smaller firms seem to have networks more centered on their region. As a whole, the traditional concepts of local innovation "milieux" are relatively difficult to apply to a region like Alsace. It is also difficult to trace a real form of transborder innovative network in the Upper Rhine valley.
\end{abstract}

\section{RESUMEN}

Este articulo trata de la nocion de una red territorial de innovacion, sobre los datos recogidos a partir de una muestra de 161 empresos con menos de 500 asalariodos, que representan el tejido industrial Alsaciano. Se pone de relieve la variedad de las redes que contribuyen al proceso de innovacion de las firmas y sobretodo la fuerte diversidad de sus proyecciones geograficas. Pero, la proximidad geografica no desempena un popel tan importante ni por las redes de participacion formal ni por las relaciones informales. Por efecto, segun el tipo de interaccion que se examina, las consecuencias de la proximidad son diversas. Ase, parece dificil hablar de un dominio o ambio de innovacion puramente Alsaciano. Para concluir, la idea de una red fronteriza entre las regiones del Rhin Superior no resulta esencial. 


\section{Introduction}

Il est particulièrement important, de nos jours, d'analyser les réseaux de partenariat ou simplement d'information qui contribuent à l'innovation des PME. Le rôle de tels réseaux est clairement reconnu dans le cas des grandes entreprises, y compris celles qui disposent en propre de départements de R-D. Ce rôle devrait être au moins aussi important pour les entreprises dont la taille réduite n'autorise pas une division du travail aussi poussée et dont on peut supposer qu'elles sont amenées à compenser le déficit de moyens internes de recherche par un recours massif (organisé ou non) aux sources externes d'idées nouvelles.

L'intérêt porté aux réseaux d'innovation des PME se trouve renforcé par la perspective régionale : la caractérisation d'un tissu industriel régional exige en effet une observation particulière des PMI, ces entreprises dont le comportement d'innovation est mal saisi au niveau des grandes enquêtes nationales, mais qui font dans une large mesure la spécificité des territoires. Les instances régionales, en particulier, ont besoin d'une analyse précise du comportement de ces entreprises et de leurs réseaux. La loi sur la décentralisation du 2 mars 1982 a légalisé les interventions économiques des régions, provoquant une explosion d'initiatives locales - bien qu'une partie des régions aient décidé de ne pas utiliser toutes les possibilités légales. D'après Lachmann (1994), on est passé d'un volume de 0,4 à 3,4 milliards de francs entre 1982 et 1990 pour les régions proprement dites et de 2,8 à 12,3 pour l'ensemble des collectivités locales. Le souci essentiel de ces interventions a toujours été le maintien de l'emploi local, mais cette politique régionale peut être qualifiée d'active: les aides aux entreprises en difficulté n'ont jamais représenté une fraction importante des interventions économiques des collectivités (elles ont même chuté de $5 \%$ en 1984 à $0,6 \%$ en 1990). La modalité d'intervention régionale est, de manière prépondérante, l'aide au développement économique et, entre autres, à l'innovation. On peut rappeler ensuite que, dans la répartition des tâches entre l'État et les régions, les collectivités locales vont être de plus en plus amenées à mettre l'accent sur le développement des PME/PMI (surtout en dessous de 250 salariés selon les nouvelles normes européennes), avec parmi les objectifs prioritaires, leur mise en réseau selon diverses formes de partenariat: entre firmes, avec des organismes parapublics, etc. Il est donc crucial pour les régions de comprendre le rôle que joue ou pourrait jouer l'interaction résiliaire des petites entreprises. Nous entendons ici par «résiliaire» une forme d'interaction de réseau, une relation qui n'appartient ni au domaine économique standard de la relation de marché, ni à celui de l'interventionnisme public classique.

En ce qui concerne les possibilités légales d'action des collectivités, il faut aussi tenir compte des contraintes de plus en strictes qu'impose l'Union 
européenne : il ne sera plus guère possible aux instances régionales d'aider à leur guise et directement les entreprises, en dehors de quelques domaines prioritaires. En fait, les seuls domaines notables d'exception seront l'environnement et l'innovation.

L'intérêt pour les réseaux régionaux d'innovation n'est pas seulement français et lié au processus de décentralisation administrative. Sur le plan théorique, l'étude des phénomènes résiliaires des PME permet d'aborder le paradoxe de la créativité de cette catégorie d'entreprises. Comme le rappelle, par exemple, Acs (1992), entre autres, à partir des travaux qu'il a réalisé avec Audretsch, le taux d'innovation (nombre d'innovations par millions d'employés) des petites firmes est plus élevé que celui des grandes. On peut argumenter sur la différence de nature et d'importance des innovations typiques de chacune des catégories d'entreprises. Mais il n'en reste pas moins que les petites font preuve d'une créativité technologique et commerciale surprenante, sachant qu'elles n'ont pas le contrôle de la variété de ressources dont profitent les grandes. Or, le phénomène ne fait que s'accentuer avec le développement régulier du poids économique des PME dans les pays occidentaux, alors même que la marche du progrès oblige de plus en plus à réaliser l'intégration complexe de technologies hétérogènes, tâche qui paraît à première vue difficile à assumer pour une entreprise de taille modeste. Une réponse naturelle est à chercher du côté des externalités : les PME doivent bien profiter de transferts de connaissances en provenance de leur environnement à travers divers réseaux. Acs (1992) évoque une forme de transfert liée au territoire (geographically mediated spillover). Les chercheurs du GREMI ont popularisé quant à eux le concept de milieu innovateur. Dans la tradition ouverte par Becattini, les spécialistes du développement technopolitain et des districts industriels examinent à un niveau très local ces effets de synergie dans des lieux géographiques précis. À l'autre extrémité du spectre géographique, les analyses consacrées aux systèmes nationaux d'innovation (Lundvall, 1988 et 1992a; Nelson, 1992) cherchent à mettre en évidence des effets de réseaux macro-économiques; or, il y a une forte interférence entre les différents niveaux géographiques. Comme le rappelle Lundvall (1992b) dans son introduction, la globalisation et la spécialisation internationale, loin de constituer une négation des environnements locaux, sont des phénomènes qui s'appuient sur des districts technologiques spécialisés et des réseaux régionaux.

La littérature actuelle sur la globalisation contredit à notre avis l'esprit du premier article qui a popularisé le mot (Levitt, 1983): les entreprises n'ignorent pas les différences régionales et nationales ou, du moins, elles peuvent à la fois jouer la carte de la commercialisation mondiale de produits standardisés et rechercher des facteurs et synergies de production ou de conception dans des environnements géographiques bien individualisés. 
Comme le montrent Cohendet et Ledoux (1994) dans le cas de la pharmacie, la globalisation porte sur certains aspects seulement de la stratégie: la recherche fondamentale à la base de l'innovation est globale, mais la recherche clinique est régionalisée. Bref, il y a place pour une forme ou une autre de territorialisation de la technologie au sein même du mouvement de globalisation. Or, les PMI constituent l'une des composantes majeures des milieux régionaux.

\section{La problématique territoriale d'une région comme l'Alsace}

En rapport avec la problématique précédemment exposée, l'observation du cas de l'Alsace nous semble particulièrement indiquée. Il est en effet intéressant de chercher à tester les modalités du comportement innovatif et la forme des réseaux des PMI dans cette région. Premièrement, parce qu'elle est fortement marquée économiquement par son tissu d'entreprises moyennes (l'Alsace possède, en comparaison nationale, des PMI aux effectifs assez étoffés et n'héberge par contre que peu de très grandes entreprises, surtout dans le domaine de la haute technologie); deuxièmement, en raison de sa situation frontalière, de son histoire et de ses spécificités socioculturelles, elle est exceptionnellement concernée par l'investissement direct international et les réseaux transfrontaliers d'échanges de produits, de technologies et de main-d'œuvre. Il y a donc particulièrement lieu de se poser, à propos d'une région comme l'Alsace, des questions précises.

- Existe-t-il une dimension locale de la technologie et comment intervient-elle dans les choix d'investissements (locaux et internationaux), ainsi que dans la productivité des firmes installées et, en particulier, des PMI qui constituent le socle du tissu industriel régional?

- Comment caractériser le ou les réseaux pertinents pour le tissu industriel d'une région frontalière comme l'Alsace: la dimension naturelle du réseau est-elle régionale, nationale, transfrontalière (bassin du Rhin supérieur) ou plus largement internationale?

- Les réseaux de l'innovation passent-ils majoritairement le long des relations de marché (apprentissage technologique interactif avec les clients, fournisseurs, équipementiers, etc.), des relations organisationnelles (transferts internes aux entreprises et aux groupes), des relations de partenariat ou, au contraire, sont-ils très informels?

- Quel rôle jouent les institutions censées assister au niveau régional les entreprises, surtout les petites, dans la maîtrise de leur environnement technico-économique? 
Outre ces questions se pose un problème de définition du champ géographique pertinent pour l'observation et l'action. Une des idées fortes de la politique technologique actuelle est, nous l'avons souligné, de renforcer le maillage des tissus d'entreprises traditionnelles régionales par des formes de partenariat entre elles et avec des organismes de transfert de technologies (centres de ressources technologiques et services d'intermédiation technologique). Mais après avoir souligné la nécessité de favoriser l'intégration résiliaire des entreprises d'un certain territoire, on ne peut pas considérer que la question soit épuisée. En effet, rien n'assure que le territoire, unité naturelle de l'intervention publique ou consulaire, puisse être posé ex ante, et donc que les réseaux pertinents pour l'entreprise aient une affinité quelconque avec un découpage géographique, ni d'ailleurs que toutes les entreprises aient pour tous leurs types de relations partenariales potentielles les mêmes structures de réseau. Les notions de proximité, de complémentarité ou de synergie dans le graphe des relations industrielles et technologiques n'ont pas de raison a priori de se traduire simplement dans la logique géographico-administrative.

Nous avons cherché à répondre à ces questions en réalisant en 1992 une enquête auprès de 200 entreprises et établissements industriels localisés en Alsace (Héraud, 1993, étude réalisée par le BETA avec le soutien de la DRIRE et de la région Alsace).

L'échantillon était représentatif du tissu régional dans sa structure par secteur d'activité et par taille (avec une limite inférieure à 20 salariés). Nous avons ainsi touché un cinquième des entreprises concernées. Dans cet article, nous exploitons un large extrait de cette base de données, soit 161 PMI pour lesquelles la question centrale du questionnaire est bien «renseignée»: celle où l'on demande aux entreprises de citer les principales sources d'information ou les partenaires ayant conduit, au cours des trois années écoulées, à une innovation ou une amélioration sensible d'un produit ou d'un procédé, en précisant la localisation de cet élément de réseau (en Alsace, dans une région transfrontalière plus large comprenant le Bade-Wurtemberg et Bâle, dans le reste de la France, le reste de l'Allemagne ou le reste du monde). Par ailleurs, un autre échantillon de 16 grandes entreprises (plus de 500 salariés) a pu être tiré de la base pour servir de point de comparaison ${ }^{1}$. Il était important de

1. Notons que nous ne disposons pas de beaucoup de grandes entreprises dans notre base globale dans la mesure où l'objectif premier de l'étude était de travailler sur un échantillon représentatif du tissu industriel alsacien et que la structure par taille impliquait de ne retenir qu'un faible nombre d'entreprises de plus de 500 salariés (suffisamment toutefois pour couvrir les principaux secteurs d'activité). En conséquence, lorsque nous donnerons des résultats moyens concernant les grandes entreprises $(\mathrm{GE})$, ce sera à titre indicatif seulement, sans grande prétention statistique. 
pouvoir néanmoins bénéficier de cette information présente dans la base de données pour remettre en perspective, au moins en ordre de grandeur, les observations faites sur les PMI.

Notre enquête cherchait fondamentalement à tester la pertinence du concept de réseau localisé d'apprentissage technologique. Les résultats, exposés plus loin, montrent une grande variété de réseaux simultanés : selon le type d'entreprise (taille ou secteur), mais aussi, pour une entreprise donnée, selon le type d'innovation ou d'amélioration de produit ou de procédé. Avant de présenter ces résultats, il nous faut développer un peu le cadre théorique qui éclaire le débat «territoire et technologie», pour mieux faire ressortir les points sur lesquels notre enquête de terrain apporte certaines réponses.

\section{PMI et systèmes localisés d'innovation}

Pour résumer en peu de mots notre problématique, nous poserons la question suivante : existe-t-il un "système d'innovation » propre à l'Alsace au sens où il serait fondé sur des réseaux fondamentalement inscrits dans le territoire régional? Notre réponse, plutôt négative, ne revient pas à nier l'existence de synergies locales ou de caractéristiques spécifiques du bassin d'emploi et du tissu industriel régional. Elle consiste seulement à récuser tout a priori du territoire.

Par exemple, et pour reprendre l'expression de Rallet (1993), les milieux innovateurs, même s'ils peuvent être constatés empiriquement, «ne sont qu'un cadre parmi d'autres, non nécessairement dominant, de la production des innovations technologiques». Il convient donc de partir des agents et de décrire leurs réseaux - dans leurs extensions géographiques fort variées - sans postuler leur nature territoriale. Il n'y a pas, au départ, de technologie alsacienne, ni même d'industrie alsacienne, mais seulement des entreprises ou établissements implantés en Alsace. Ce n'est qu'après observation détaillée qu'il sera possible de dégager d'éventuels réseaux qui «bouclent » ou «font système » en région. Nous vérifierons alors si certains réseaux font exister un territoire régional.

Dans le domaine de l'innovation, la dimension partenariale de l'activité économique est particulièrement présente. Ce fait n'est pas nouveau et a été souvent rappelé (voir les divers modèles de l'apprentissage par l'interaction avec les utilisateurs, par l'information technologique incorporée dans les équipements, par échange réciproque d'actifs immatériels complémentaires, etc.). Si les réseaux d'innovation ont toujours existé, une évolution est cependant sensible dans leur forme. Freeman (1991) rappelle que ce qui est relativement nouveau à partir des années 80 , c'est le développement d'accords de coopération explicites. L'auteur propose de distinguer clairement ces types de partenariat et, à la suite de Camagni, préfère réserver le 
terme de réseau aux accords explicites (juridiques). Ces accords constituent des liens préférentiels engageant explicitement les partenaires, dans l'objectif de réduire l'incertitude sur la technologie, les marchés, etc. Dans cette catégorie se retrouvent les entreprises (joint venture), les accords de R-D et les licences d'exploitation, la coopération avec le secteur public, etc. Par ailleurs, le terme de milieu pourrait être consacré aux types informels de relations externes et à l'échange tacite (non codifié) d'informations. Freeman précise à ce propos que les milieux innovateurs présentent assez souvent une dimension locale. On peut faire rentrer dans cette catégorie ces relations informelles entre individus dont Von Hippel (1988) a souligné l'importance dans les processus d'innovation et que plusieurs auteurs, traitant de la relation technologie-territoire, présentent comme une source essentielle de compétitivité des milieux locaux.

Du point de vue des PME se pose alors la question suivante: sont-elles capables de relations partenariales construites et d'alliances aussi «stratégiques» que les grandes entreprises (formant ainsi des réseaux au sens strict indiqué ci-dessus), ou bien sont-elles principalement impliquées dans des relations de milieu, largement informelles et localisées? À la notion de partenariat est associée une certaine forme de confiance (à la différence de la relation hiérarchique fondée sur le pouvoir ou de la relation de marché éphémère et opportuniste). Bon nombre d'auteurs intéressés par la dialectique technologie/territoire ont fait l'hypothèse que la proximité géographique facilitait ce type de rapports. On aurait alors tendance à penser que la spécificité des petites entreprises constituant le tissu régional est de tisser des liens technologiques sur le mode de la relation informelle et de proximité.

Ce qui nous intéressera particulièrement de tester sur notre échantillon de PMI alsaciennes est de savoir dans quelle mesure leurs relations partenariales, et plus généralement leurs comportements résiliaires, sont plus ou moins liés à un concept de proximité. Notre argument est qu'une partie de la littérature économique, par exemple, dans la filiation des districts industriels introduits par Marshall et repris par l'école italienne à la suite de Becattini (1979), a eu tendance à surestimer quelque peu la composante locale de la relation de confiance.

Il est certain qu'un territoire aussi localisé que le «district» autorise d'importantes économies d'information et donc de coûts de transaction. De plus, il se fonde sur une histoire et une culture locales impliquant proximité culturelle, limitation de l'opportunisme et communication informelle renforcée. Ainsi, comme le rappelle Veltz (1991) en évoquant les analyses de Piore et Sabel (1984), le district industriel marshallien ou «à l'italienne», qui est l'exemple même du réseau fortement territorialisé, est une forme de 
partenariat de PME où la relation de confiance relève de traditions et de comportements bien établis, de nature largement extra-économique, qui l'empêchent de dégénérer en concurrences internes meurtrières. Ce type de réseau partenarial est effectivement fortement inscrit dans un territoire, car fondé sur l'histoire de ce territoire. Mais à l'époque de la mondialisation des flux économiques et de la technologie (que les nouvelles techniques de transport et de communication apportent ou imposent aussi aux petites entreprises), le facteur de proximité géographique joue un peu moins. Pour ne prendre que l'exemple des relations informelles entre cadres, l'expérience montre qu'elles se nouent autour de réseaux interpersonnels que la distance n'abolit pas. La proximité des acteurs est certes un facteur fondamental facilitant la constitution des réseaux, mais d'autres concepts de proximité que celui de l'espace ordinaire doivent être envisagés (et peut-être même par priorité): proximités culturelle, technologique, organisationnelle, etc.

Des éléments envisagés par Marshall (1920), comme fondement des spécificités locales, la plus durable nous semble être le marché du travail. En effet, la main-d'œuvre reste largement fixée dans des bassins d'emploi et constitue en quelque sorte le support le plus localisé des apprentissages collectifs générés et utilisés par les firmes. C'est pourquoi nous croyons principalement aux vertus des politiques régionales en matière de formation. (Dans le cas de l'Alsace, le régime local particulier de l'apprentissage renforce encore la spécificité régionale.) Pour les autres facteurs, nous verrons que la dimension régionale est bien présente, mais de manière inégale selon le type de relation résiliaire chez les PMI alsaciennes.

Cela dit, le territoire ne disparaît pas pour autant dans toutes ses autres dimensions. Il n'existe pas ex ante, mais apparaît comme produit des stratégies des acteurs. Ce concept dynamique de territoire est maintenant largement établi. Par exemple, Perrin (1990) pose une définition du district comme un espace dans lequel prend place une procédure d'apprentissage collectif. Bref, le territoire ainsi défini est le lieu de matérialisation d'avantages construits par un certain réseau d'acteurs. C'est ce que nous voulons exprimer (sans nous limiter au cas du «district», qui n'était pris ici que comme exemple analytique, et sans prétendre ramener à lui toute problématique territoriale) en disant que le territoire ne doit pas être donné au départ de l'analyse économique, mais observé comme un produit des stratégies d'acteurs et des réseaux que ces derniers construisent. Seuls les acteurs institutionnels et leurs politiques sont localisés a priori.

Comme l'exprime très bien Rallet (1993), il ne faut pas confondre «lieu» et «territoire». Les acteurs sont d'un lieu : par exemple, dans le cas qui nous intéresse, des entreprises ou des établissements ont une adresse en 
Alsace. Mais ils n'appartiennent pas nécessairement à un «territoire »: il n'y a pas a priori une industrie alsacienne. D'autres études que nous avons réalisées sur le tissu industriel alsacien (Héraud et Nanopoulos,1994) confirment la forte disparité des entreprises et des établissements situés en région: il y a les vraies et les fausses PMI régionales (il convient d'observer les liaisons financières), les firmes qui utilisent beaucoup et celles qui n'utilisent jamais le système d'aide régional, etc. Par ailleurs, le réseau de la soustraitance est apparu fortement éclaté entre le nord et le sud de la région. Nous sommes, en effet, arrivés à la conclusion qu'en matière de sous-traitance, l'Alsace est constituée de deux districts industriels, respectivement centrés sur Strasbourg et Mulhouse, qui ont chacun plus de rapports avec l'extérieur de la région qu'entre eux. Le cas des réseaux de sous-traitance illustre bien la spécificité de la notion de proximité, selon le point de vue économique. Les commentaires d'industriels donneurs d'ordres nous font penser que la distance, pour eux, est en quelque sorte une variable à deux modalités. Grossièrement, si le contact matériel avec le sous-traitant ne peut se faire en moins d'une demi-journée aller-retour, il sera considéré comme éloigné (et dans ces conditions l'appartenance à la région importe peu). Pour toute étude territoriale, il faut donc, à notre avis, commencer par réaliser une typologie de firmes en fonction de leurs réseaux et juger ensuite de la territorialité des acteurs et des relations.

Il nous reste maintenant à décrire les principaux résultats de notre enquête selon une grille de lecture inspirée des considérations théoriques précédentes.

\section{Les réseaux technologiques et économiques des PMI en Alsace}

Notre enquête a principalement permis de retracer le comportement d'innovation des entreprises régionales. Les questions ne portaient pas frontalement sur l'effort de recherche. Nous avons, cependant, un minimum d'information sur la R-D effectivement pratiquée. Comme on pouvait s'y attendre, notre échantillon de PMI fait peu de recherche au sens des statistiques officielles. À l'aune de la définition du manuel de Frascati, tout se passe comme si les entreprises innovaient sans avoir vraiment fait de recherche! En fait, ce qui apparaît là clairement, c'est le problème de la mesure de l'effort d'investissement technologique (et plus généralement d'ailleurs de l'investissement immatériel) des petites entreprises. Ainsi, dans notre échantillon, bien que les trois quarts des entreprises déclarent avoir une «fonction R-D »; moins du tiers emploient au moins une personne à temps complet à des activités de type études et méthodes. 
Parmi les formes de réseau d'innovation qui se dégagent de notre enquête, la majorité concernent des effets de «milieu» (relations technologiques parallèles aux échanges avec les clients et les fournisseurs, contacts informels, imitation, etc.). C'est principalement sur cet ensemble d'informations, présenté dans le tableau 1 (description des sources externes de l'innovation), que nous pouvons analyser les réseaux d'innovation d'où les entreprises régionales tirent leurs idées d'innovation ou d'amélioration de produits ou de procédés.

\subsection{La nature plus ou moins formelle des réseaux d'innovation}

Sauf exception (les PME de haute technologie), la source habituelle de l'innovation ne peut pas être, pour une petite entreprise, la R-D interne au sens d'un effort structuré et régulier de recherche technologique. Mais il faut souligner également que les relations résiliaires qui s'y substituent ne sont pas majoritairement de type formel. Pour être tout à fait précis sur ce point, il convient de faire la distinction entre trois niveaux de liaisons:

- Les accords de coopération sont l'expression parfaite d'une relation formelle. Sur 161 PMI, moins de la moitié (69) ont déclaré l'une ou l'autre forme d'accord (sur une période de trois ans). Et sur cet ensemble, les accords de R-D représentent moins du tiers (28\%).

- Les relations diverses qui forment l'essentiel de la base de données (voir tableau en annexe) ne sont pas dans leur grande majorité formalisées juridiquement. Les 161 PMI de notre échantillon ont déclaré au total 1241 relations.

- Parmi ces relations, $7 \%$ ont été citées sous la rubrique «contacts informels ».

En ce qui concerne les réseaux formels, les 69 PMI concernées ont déclaré 196 accords de coopération dont 57 en R-D ( 35 pour des produits et 22 pour des procédés); les autres concernant la commercialisation (71), la production (55) et la finance (13).

Il ressort donc de notre enquête qu'un bon nombre de PMI alsaciennes pratiquent une forme de recherche (et pensent que la R-D est stratégique), même lorsqu'elles n'ont pas de structure permanente de R-D. Les chiffres montrent qu'elles n'ont guère tendance à compenser un défaut de capacité de R-D par un recours massif à la recherche externe que peuvent fournir les centres de recherche et consultants privés ou les organismes publics. Quant au partenariat de R-D dans sa version la plus formalisée, les accords de coopération, il n'apparaît qu'à partir d'un seuil de taille très marqué : 
approximativement, au-dessus de 300 employés. En gros, ce sont les entreprises moyennes qui commencent à être vraiment actives en matière de réseau d'innovation, si l'on prend ce terme au sens fort d'un partenariat officiel.

Il est intéressant d'essayer de comparer les comportements de partenariat formel en R-D des PMI aux comportements des GE sur ce terrain qui est beaucoup plus naturel pour les secondes. Le faible nombre de GE dans notre échantillon ne nous permet pas une comparaison très significative, mais on peut constater qualitativement que la proportion d'accords de R-D est plus forte ( $40 \%$ contre $28 \%$ ) et que c'est la R-D de procédé qui est de loin la plus importante dans cette catégorie (au contraire des PMI).

On peut aussi chercher à comparer les réseaux formels des PMI à leurs réseaux au sens large. Nous donnons ci-dessous la répartition géographique des accords de R-D de produit (statistique sur 35 accords) en comparaison avec l'ensemble des relations citées (1 242 cas):

TABleau 1

\begin{tabular}{l|c|c}
\hline \multicolumn{1}{c|}{$\begin{array}{c}\text { Zone géographique } \\
\text { \% }\end{array}$} & Accords formels & Total des relations \\
\hline Alsace & 26 & 27 \\
Bade-Wurtemberg & 3 & 5 \\
Bâle & 0 & 2 \\
Reste de la France & 40 & 38 \\
Reste de l'Allemagne & 14 & 14 \\
Reste du monde & 17 & 14 \\
Total & $\mathbf{1 0 0}$ & $\mathbf{1 0 0}$ \\
\hline
\end{tabular}

On voit que la répartition n'est pas significativement différente, si ce n'est un léger biais en défaveur du local pour les réseaux formels. Mais on ne peut pas prendre de tels résultats pour preuve décisive de la dimension locale des relations les moins formelles.

Il nous reste à analyser un dernier niveau, celui des relations déclarées comme étant des «contacts informels», c'est-à-dire des sources externes d'idées d'innovations qui ne correspondent pas à un partenaire au statut précis ou à une source d'information standard.

Les relations informelles de ce type constituent une partie non négligeable des relations conduisant à l'innovation des PMI. Elles correspondent en effet à $7 \%$ des citations (presque autant à elles seules que toutes les relations avec les institutions qui atteignent $9 \%$ ). Mais il ne faut pas exagérer 
leur rôle, puisque ce chiffre reste très inférieur au poids des relations avec la clientèle $(17 \%)$, aux sources documentaires et expositions $(18 \%)$ ou à l'ensemble des fournisseurs (24\%).

Cette observation concernant les PMI doit être, de plus, comparée à celle des GE. Curieusement, dans notre échantillon, l'informel semble tenir plus de place dans les entreprises et établissements de plus de 500 salariés : $10 \%$ contre $7 \%$. On ne peut donc pas accepter le modèle de la petite entreprise dépendant plus particulièrement des relations technologiques informelles.

Par contre, ces contacts informels sont plus nettement situés en région pour les PMI (34\%) que pour les GE (20\%). Il est, toutefois, difficile de parler d'une forte connexité du graphe des relations informelles des PMI régionales et donc de démontrer à travers nos mesures l'existence d'un modèle fort de «milieu innovateur». En effet, on observe au moins autant d'extension nationale ( $35 \%$ hors Alsace) que régionale pour le réseau informel. Il serait plus juste de dire, a contrario, que c'est l'ensemble des GE qui présente une forte extraversion des contacts informels (dont $33 \%$ dans le reste de la France et $47 \%$ à l'étranger).

\subsection{Analyse de l'effet de proximité}

Nous allons poursuivre l'analyse des résultats sur ce thème de la répartition géographique des réseaux en abordant globalement la question de la localisation des partenaires et des sources d'information, c'est-à-dire en exploitant la marge inférieure du tableau donné en annexe.

Sur les 1241 liaisons citées par les PMI, 334 se situent en Alsace. Le total des liaisons avec la France (Alsace comprise) est de 805. On peut donc construire, en rapportant ces deux derniers chiffres, un indice de concentration régionale du réseau global des PMI alsaciennes, que nous appellerons RES(ALS/FRA): il vaut $34 \%$.

Dans le cas des GE régionales, notre échantillon conduit à estimer la valeur de cet indice à $27 \%$. Compte tenu de la faiblesse relative de l'échantillon des GE (16 entreprises citant 147 relations), on ne peut pas tirer de conclusion définitive. L'hypothèse d'une plus forte «régionalité » des réseaux des PMI ne peut pas être rejetée, mais elle n'est pas non plus très fortement confortée par notre mesure.

Une autre manière de tester l'influence de la proximité géographique dans la construction des réseaux consiste à considérer le couple BadeWurtemberg/Allemagne que nous noterons RES(BW/RFA). Dans ce cas, on n'observe aucune différence statistique notable entre PMI et GE puisque 
les valeurs sont respectivement de $27 \%$ et $26 \%$. Il est donc impossible de conforter le modèle du tissu régional de PMI plus particulièrement ancré sur l'espace transfrontalier du Rhin supérieur.

Pour mieux cerner l'effet de proximité, on peut chercher à relativiser l'information procurée par ces indices de concentration de réseaux en les rapportant à l'importance économique des zones géographiques considérées. Ainsi, les $37 \%$ de relations tissées en Alsace peuvent être comparées aux $3 \%$ que représente le poids économique de l'Alsace dans l'ensemble national calculé en termes de revenu national, soit PIB(ALS/FRA). De même, les $27 \%$ de concentration sur le $\mathrm{BW}$ sont à comparer au poids du BW dans l'Allemagne entière (hors nouveaux Länder), soit $16 \%$. L'ensemble de ces chiffres est résumé dans le tableau suivant. On peut conclure à un effet de proximité non négligeable en Alsace même, mais nettement plus limité dans la dimension transfrontalière, ce qui tend à montrer que la distance institutionnelle joue un rôle plus important que la distance géographique.

TABleau 2

\begin{tabular}{l|c|c|c}
\hline & $\begin{array}{c}\text { Réseau } \\
\text { PMI }\end{array}$ & $\begin{array}{c}\text { Réseau } \\
\text { GE }\end{array}$ & $\begin{array}{c}\text { Poids économique } \\
\text { des régions }\end{array}$ \\
\hline $\begin{array}{l}\text { Alsace/France } \\
\begin{array}{l}\text { Bade-Wurtemberg/République } \\
\text { fédérale allemande }\end{array}\end{array}$ & 0,41 & 0,34 & 0,03 \\
\hline
\end{tabular}

\subsection{Analyse des réseaux par type de partenaire}

Les interlocuteurs les plus souvent cités sont, comme on le voit sur le tableau en annexe, les clients et les fournisseurs (équipementiers et autres). Les contacts noués à l'occasion de foires et d'expositions professionnelles constituent également une importante source d'informations, pour les entreprises de l'échantillon.

Les autres interlocuteurs et sources d'informations cités ensuite sont essentiellement les entreprises du même secteur, les contacts informels et les sources bibliographiques. On peut aussi considérer comme important, de manière agrégée, l'ensemble des liens organiques (relations avec les autres établissements de la société, la maison mère et les autres sociétés du groupe).

Par contre, les centres de recherche privés et les organismes publics d'aide à la création et au transfert de technologie font partie des partenaires les moins cités. Ce dernier point mérite un rapide commentaire, en relation 
avec les résultats d'études menées en région par le BETA (voir Cohendet et Llerena, 1992): lorsqu' on cherche à évaluer le rôle de partenaires comme les CRITT (Centre régionaux d'innovation et de transfert de technologie), on peut dresser un bilan tout à fait positif de leur rôle en ce qui concerne les entreprises susceptibles d'être intéressées par le champ technologique précis de chacun des CRITT, mais il faut bien être conscient que seule une petite partie du système technique global de l'industrie est couverte par l'ensemble des CRITT créés en région. C'est d'autant plus vrai en Alsace que les CRITT y sont relativement spécialisés.

Outre les relations informelles que nous avons analysées en 3.1., quatre catégories de relations méritent que l'on s'attarde à les examiner avec plus d'attention.

\subsubsection{La relation avec la clientèle et les fournisseurs}

Une proportion importante $(17 \%)$ des citations concerne une relation avec un client. Ce que l'on appelle classiquement l'apprentissage par l'usage (Rosenberg, 1982) semble donc bien être l'un des schémas d'innovation dominants pour les petites entreprises, ce qui est cohérent avec le fait qu'elles réalisent particulièrement des innovations de produit. Il faut noter que ce score est du même ordre de grandeur que celui des grandes entreprises $(15 \%)$. La principale différence entre ces deux catégories d'entreprises est que les PMI développent plus cette forme d'apprentissage par interaction en région que les GE (respectivement $24 \%$ et $9 \%$ des relations avec la clientèle). Mais dans les deux cas, c'est l'espace national qui est la référence dominante (39\% et $36 \%$ ).

Les fournisseurs d'équipements procurent également un nombre important d'occasions d'innovation, avec $14 \%$ de citations des PMI (12\% pour les GE). Les PMI vont beaucoup plus chercher ces fournisseurs en Alsace $(20 \%)$ et dans le reste de la France (34\%) que les GE (respectivement $6 \%$ et $17 \%$ ). Comme on pouvait s'y attendre, les fournisseurs d'équipement allemands jouent un rôle important, même pour les PMI ( $28 \%$ de citations, dont $8 \%$ pour le Bade-Wurtemberg).

Les autres fournisseurs (produits intermédiaires, matières premières et énergie, etc.) jouent un rôle presque aussi important (10\%) dans les réseaux d'innovation des PMI et ils sont un peu plus concentrés en région ( $26 \%$ du total de ces relations de fournisseurs). 


\subsubsection{La relation organique: les contacts intragroupe}

Nous réunissons sous l'appellation «intragroupe» les contacts entre établissements de la même entreprise, entre entreprises du même groupe et entre filiales et maison mère. Ce type de relations représente $10 \%$ des réseaux d'innovation des PMI alsaciennes (contre $15 \%$ pour les établissements ou entreprises de plus de 500 salariés). Ce réseau est concentré en Alsace à hauteur de $24 \%$. Le reste de la France compte beaucoup (32\%) de même que l'Allemagne (23\%, dont $12 \%$ pour le seul Bade-Wurtemberg).

Ce domaine est le seul où la proximité transfrontalière joue un rôle notable, en particulier pour ce qui concerne la relation avec la maison mère: le Bade-Wurtemberg représente $17 \%$ du réseau organique de ce type (alors que son poids n'est que de $5 \%$ dans le total des relations citées). C'est également dans ce domaine qu'apparaît l'un des meilleurs scores de la région de Bâle (5\% contre $2 \%$ du poids global dans les réseaux des PMI alsaciennes). Au total, on peut affirmer que la principale relation technologique transfrontalière est celle qui lie des maisons mères badoises ou bâloises à des filiales alsaciennes. Notons que l'on retrouve exactement le même schéma pour les unités de plus de 500 salariés.

\subsubsection{Les relations institutionnelles}

L'ensemble des relations institutionnelles - organismes consulaires, centres technologiques professionnels, organismes publics d'aide à l'innovation et au transfert de technologies, universités et grands organismes publics de recherche - constituent seulement $9 \%$ du réseau innovant des PMI. Notons que c'est presque exactement la même proportion que pour les GE. Le fait le plus marquant concernant le réseau institutionnel est qu'il est fortement centré sur la région (55\%). Il s'agit là du plus fort indice de régionalisation, devant l'ensemble des consultants et des centres de recherche privés $(52 \%)$. On peut présenter ce phénomène en parlant d'une attitude de recherche de «guichets en région».

Il est remarquable à ce propos de noter qu'aucun contact avec l'université et la recherche publique n'est cité au Bade-Wurtemberg (contre 17 en Alsace), malgré la présence à proximité d'universités techniques et de pôles scientifiques de renom à Karlsruhe et à Stuttgart. L'institutionnel appartient donc clairement bien plus à une logique territoriale administrative qu'à une proximité géographique.

Les grandes unités font un peu moins appel, proportionnellement, aux «guichets de proximité » $(43 \%)$. Outre le fait que leurs réseaux sont plus mondialisés, il faut noter qu'elles ne sont pas admissibles à certains types 
d'aide publique régionale. Par contre, notre échantillon fait apparaître un tiers de relations institutionnelles avec l'Allemagne, dont la moitié au BadeWurtemberg. Il y a donc un contraste total avec les PMI pour lesquelles le transfrontalier est particulièrement absent de la relation institutionnelle.

L'espace du Rhin supérieur est encore bien loin de jouer un rôle institutionnel significatif, alors que le Bade-Wurtemberg, par exemple, est justement réputé pour son système d'aide au transfert de technologies, lequel a contribué au développement du Mittelstand (ce tissu de PME solides et actives qui fait la spécificité - et la fierté - du Land). Le partenariat institutionnel des petites entreprises alsaciennes reste fondé sur des réflexes nationaux, avec un recours quasi automatique aux représentants locaux de l'administration et à leurs organisations professionnelles les plus proches.

\subsubsection{Autres relations}

Soulignons pour finir deux types de relations où le réseau d'innovation des PMI alsaciennes n'apparaît pas très régional. Dans le cas de l'imitation ou du partenariat avec des entreprises du même secteur, l'Alsace ne représente que $21 \%$ (contre $46 \%$ dans le reste de la France et $33 \%$ à l'étranger). Bâle et le Bade-Wurtemberg comptent également peu ( $3 \%$ ensemble). L'autre domaine est l'ensemble important des sources d'informations organisées (foires, expositions, documentation écrite ou électronique). Cette partie du réseau d'innovation des PMI alsaciennes a un poids important $(18 \%)$, supérieur à ce qu'il est pour les GE (13\%). Il est très réparti géographiquement, avec $44 \%$ dans le reste de la France, $24 \%$ dans l'ensemble de l'Allemagne (dont seulement $4 \%$ en Bade-Wurtemberg) et $19 \%$ dans le reste du monde. L'Alsace ne compte que pour $12 \%$. Cette répartition géographique étendue n'est pas pour nous étonner, mais elle est importante à noter du fait qu'au total ce type d'information joue un rôle de premier plan dans l'innovation des PMI alsaciennes: deux fois plus que l'ensemble de toutes les relations institutionnelles.

\subsection{Analyse synthétique de la projection géographique des réseaux}

Une manière commode de présenter synthétiquement la «carte» des différents réseaux d'innovation des PMI alsaciennes consiste à partir d'une analyse factorielle des correspondances réalisée sur le tableau des 1241 citations (voir figure 2) 
Le premier axe factoriel ( $66 \%$ de l'inertie totale) oppose très nettement le proche au lointain: la localisation en Alsace d'un côté et à l'étranger de l'autre, avec les liaisons nationales (reste de la France) en position neutre, c'est-à-dire proche de l'origine, au milieu de l'axe. En ce qui concerne la projection des points «partenaires » sur le même axe, on peut y lire la confirmation du fait que c'est le partenariat institutionnel qui est le plus typiquement associé à la dimension régionale. Il convient seulement d'ajouter à ce réseau de proximité les consultants et les centres de recherche privés. Au contraire, les fournisseurs d'équipement et les contacts établis lors de foires et d'expositions se réalisent en moyenne à bonne distance.

Le deuxième axe (19\% de l'inertie) oppose la localisation transfrontalière (Bade-Wurtemberg et Bâle) à toutes les autres localisations; et ce sont les relations avec la maison mère du groupe qui constituent le seul point «partenaire» qui se détache fortement dans cette direction.

Le tableau 3 agrège les réponses de 161 PMI à la question concernant les interlocuteurs ou les sources d'information qui ont contribué, au cours des années 1990 à 1992, au développement ou à l'introduction de produits techniquement améliorés ou à l'introduction de produits ou procédés nouveaux. Chaque chiffre correspond au nombre de citations de telles relations (au total 1241 ) dans la zone géographique indiquée en colonne.

En résumé, la territorialisation suivante des réseaux technico-économiques des PMI alsaciennes peut être proposée.

- Le réseau des partenaires institutionnels est principalement situé en région; il implique particulièrement les organismes professionnels, les centres de transfert de technologie et la recherche publique. On peut aussi raccrocher à ce système régional les consultants privés. Si ce réseau «alsacien » est assez clairement défini, il n'est pas cité, au total, de manière massive parmi les facteurs ayant contribué à l'innovation.

- Le reste de la France constitue un système fort où s'enracinent particulièrement les relations avec les clients, les contacts informels et les fournisseurs (hors équipement).

- Le pôle étranger est dominé par l'Allemagne. Il se caractérise par l'importance des relations avec les fournisseurs d'équipement et des contacts dans les foires et les expositions. Dans une moindre mesure, on y trouve également les réseaux organisationnels (autres entreprises du groupe). On peut affiner l'analyse de l'étranger en signalant que le réseau des fournisseurs d'équipement, de même que celui des expositions et des foires est assez caractéristique de l'Allemagne, alors que les liaisons avec les entreprises du même groupe industriel apparaissent plus mondialisées. 
Tableau 3

Les sources externes de l'innovation des PMI alsaciennes

\begin{tabular}{|c|c|c|c|c|c|c|c|c|}
\hline Nombre de citations de relations & Alsace & $\begin{array}{c}\text { Bade- } \\
\text { Wurtemberg }\end{array}$ & Bâle & $\begin{array}{l}\text { Reste de la } \\
\text { France }\end{array}$ & $\begin{array}{c}\text { Reste de } \\
\text { l'Allemagne }\end{array}$ & $\begin{array}{c}\text { Reste du } \\
\text { monde }\end{array}$ & Total & $\%$ \\
\hline Autres établissements de l'entreprise & 10 & 1 & 1 & 6 & 1 & I & 20 & 1,61 \\
\hline Maison mère du groupe & 14 & 10 & 3 & 17 & 6 & 8 & 58 & 4,67 \\
\hline Autres entreprises du groupe & 7 & 4 & 2 & 18 & 6 & 12 & 49 & 3,95 \\
\hline Fournisseurs d'équipement & 35 & 13 & 3 & 58 & 35 & 29 & 173 & 13,94 \\
\hline Autres fournisseurs & 34 & 4 & 6 & 46 & 21 & 15 & 126 & 10.15 \\
\hline Clients & 52 & 16 & 8 & 82 & 24 & 31 & 213 & 17,16 \\
\hline Entreprises du secteur & 20 & 2 & 1 & 44 & 11 & 17 & 95 & 7,66 \\
\hline Entreprises d'autres secteurs & 11 & 0 & 0 & 9 & 3 & 3 & 26 & 2,10 \\
\hline Cabinets de consultants & 25 & 1 & 0 & 17 & 0 & 1 & 44 & 3,55 \\
\hline Centres de recherche privés & 8 & 0 & 0 & 7 & 1 & 3 & 19 & 1,53 \\
\hline $\mathrm{CCl}$, organisations professionnelles & 18 & 0 & 0 & 10 & 0 & 2 & 30 & 2,42 \\
\hline Centres techniques professionnels & 13 & 0 & 0 & 14 & 1 & 1 & 29 & 2,34 \\
\hline $\begin{array}{l}\text { Universités, organisations publiques } \\
\text { de recherche }\end{array}$ & 17 & 0 & 0 & 11 & 1 & 3 & 32 & 2,58 \\
\hline Expositions, foires & 15 & 7 & 1 & 59 & 57 & 28 & 147 & 11,85 \\
\hline Contacts informels & 29 & 4 & 3 & 30 & 11 & 9 & 86 & 6,93 \\
\hline Bibliographie, banques de données... & 12 & 1 & 1 & 37 & 8 & 14 & 73 & 5,88 \\
\hline Total & 334 & 63 & 29 & 471 & 167 & 177 & 1241 & 100,00 \\
\hline$\%$ & 26,91 & 5,08 & 2,34 & 37,95 & 13,46 & 14,26 & 100,00 & \\
\hline
\end{tabular}


- Enfin, le quatrième système repéré par cette analyse de données est celui de l'espace transfrontalier caractérisé principalement par les rapports avec la maison mère.

Il apparaît nettement qu'il est difficile de parler de l'industrie alsacienne et de ses réseaux comme si le territoire alsacien était donné et qu'il impliquait un certain déterminisme sur les entreprises qui s'y trouvent localisées. Sur le plan méthodologique, il faut repartir des comportements individuels des sujets, observer leurs réseaux et bâtir des regroupements plus homogènes dont on peut alors caractériser les espaces pertinents.

\section{Conclusion}

Notre enquête a montré qu'au sein d'une économie régionale comme l'Alsace, presque tous les schémas d'innovation et de diffusion du progrès technique que distingue la littérature (Rosenberg, 1982; Pavitt, 1986; Dosi, 1988 et Von Hippel, 1988) peuvent être illustrés : diverses formes d'apprentissage par interaction, transfert par l'équipement, contacts informels, partenariat privé ou public, etc. À chacun d'eux, correspond un réseau original en termes de localisation comme de type partenaire. En raison de cette grande variété des réseaux d'innovation des PMI, on ne peut pas faire apparaître une dimension régionale systématique. On observe au contraire un éclatement géographique des réseaux et un impact assez modéré du facteur de proximité géographique. Il est significatif d'observer que le type d'interaction qui apparaît le plus systématiquement enraciné dans le territoire régional est de nature institutionnelle. Autrement dit, plus qu'un fonctionnement de milieu régional, ce que traduisent une majeure partie des relations en région, c'est une logique de guichet de proximité. Cette proximité est bien administrative: la partie transfrontalière de la zone de proximité de l'Alsace est très peu sollicitée pour ces contacts institutionnels (les guichets doivent être dans l'Hexagone). Par contre, le Bade-Wurtemberg est très présent dans l'échange interne des groupes industriels, les filiales alsaciennes bénéficiant d'importants transferts.

Notre étude des réseaux d'innovation des PMI alsaciennes a confirmé la difficulté de rendre compte d'un milieu régional de manière simple. Mais surtout elle montre, nous semble-t-il, que le territoire ne doit pas être posé ex ante dans une analyse des réseaux d'entreprises. Il convient d'approcher le comportement résiliaire des acteurs sans a priori géographique autre qu'administratif : le territoire n'est donné que pour les institutions qui l'ont en charge. L'entreprise, elle, construit son territoire pertinent en tissant ses réseaux, c'est-à-dire en développant sa stratégie propre. Et l'ensemble des 
entreprises d'une région (fussent-elles des petites entreprises) n'a pas de raison a priori de présenter une cohérence géographique notable de ses réseaux. En d'autres termes, la relation technologie-territoire, y compris pour les PME, ne suit pas nécessairement le paradigme du district industriel, si l'on entend par là un territoire où se manifeste une surdétermination forte et convergente des comportements résiliaires de tous les acteurs.

Mais on peut aussi tirer des observations faites sur le terrain alsacien un autre type de conclusion, à savoir que cette région, malgré sa relative bonne santé économique, présente un certain manque de cohésion technologique et, plus largement, de cohésion stratégique. De ce point de vue, le fait que les réseaux soient assez diversement déployés pourrait être interprété comme un signe de dépendance, comme la manifestation du fait qu'elle n'appartient pas au cœur d'une zone de leadership technologique, mais qu'elle y est seulement rattachée. Bien entendu, aborder une telle question suppose un travail d'analyse qui dépasse le cadre du présent article.

\section{Bibliographie}

ACS, Z.J. (1992), «Small business economics : a global perspective», Challenge, novembre-décembre.

BECATtini, G. (1979), «Dal settore industriale al distretto industriale : alcune considerazioni sull'unità di indagine dell'economia industriale », Rivista di economia politica industriale, $\mathrm{n}^{\circ} 1$.

Cohendet, P. et P. Llerena (1992), «Évaluation de la politique en faveur du transfert de technologie : le cas des CRITT en Alsace», Rapport d'étude pour la DRRT, la DATAR et la Région Alsace, BETA, Strasbourg.

Cohendet, P. et M.J. Ledoux (1994), «The chemical industry towards globalisation », dans U. Muldur et R. Petrella (dir.), Science and Technology Policy, Bruxelles, Commission des Communautés européennes, EUR 15150.

DosI, G. (1988), « Sources, procedures and microeconomic effects of innovation», Journal of Economic Literature, vol. 26, septembre, p. 1120-1171.

FreEman, C. (1991), «Networks of innovators : a synthesis of research issues », Research Policy, ${ }^{\circ} 20$, p. 499.

HÉRAUD, J.A. (1993), Les réseaux économiques et technologiques de l'industrie alsacienne, Étude réalisée avec le soutien du Conseil régional et de la DRIRE d'Alsace, BETA, ULP, Strasbourg.

HÉRAUD, J.A et K. NANOPOULOS (1994), La sous-traitance industrielle en Alsace, Étude réalisée pour le compte du Conseil régional d'Alsace, BETA, Université Louis-Pasteur, Strasbourg. 
HIPPEL, E. VON (1988), The Sources of Innovation, New York, Oxford University Press.

LACHMANN, J. (1994), «L'inflexion des politiques économiques territoriales », Les Cahiers du CNFPT, Centre national de la fonction publique territoriale, $n^{\circ} 40$, mars, p. 202-210.

LEVITT, T. (1983), «The globalization of markets », Harvard Business Review, mai-juin.

LUNDVALL, B.A. (1988), «Innovation as an interactive process : from user-producer interaction to the national system of innovation», dans Dosi et al. (1988).

LUNDVALL, B.A. (1992a), «Relations entre utilisateurs et producteurs, systèmes nationaux d'innovation et internalisation », dans D. Foray et C. Freemann (dir.), 1992.

LundVall, B.A. (dir.) (1992b), National Systems of Innovation. Towards a Theory of Innovation and Interactive Learning, Londres, Pinter Publ.

Marshall, A. (1920), Principles of Economics, Londres, The McMillan Press Ltd.

NELSON, R.R. (1992), « National innovation systems : retrospective on a study », Institutional and Corporate Change, vol. $1, \mathrm{n}^{\circ} 2$, p. 347-374.

PAVITT, K. (1986), «Patterns of technical change : towards a taxonomy and a theory », Research Policy, vol. 13, p. 343-373.

PERRIN, J.C. (1990), «Organisation industrielle: la composante territoriale», Revue d'Économie Industrielle, $\mathrm{n}^{\circ} 51$, premier trimestre.

PIORE, M.J. et C.F. SABEL (1984), The Second Industrial Divide: Possibilities for Prosperity, New York, Basic Books.

RALlET, A. (1993), «Choix de proximité et processus d'innovation technologique », Revue d'Économie Régionale et Urbaine, $\mathrm{n}^{\circ} 3$.

Rosenberg, N. (1982), Inside the Black Box: Technology and Economics, Cambridge, Cambridge University Press.

Veltz, P. (1991), «Communication, réseaux et territoire dans les systèmes de production modernes », dans F. Rove F. et P. Veltz, Entreprises et territoires en réseaux, CNRS Éditions. 